\title{
Knowledge and Awareness of Brucellosis among Pregnant Women in Yemen
}

\author{
Al-Nowihi $\mathbf{M}^{1 *}$, Al-Arnoot S1,2, Abdullah QYM$^{1}$ and Al-Thobhani $\mathrm{A}^{1}$ \\ ${ }^{1}$ Department of Biology, Sana'a University, Yemen \\ 2Microbiology Department, Global Pharma Co Ltd, Yemen
}

*Corresponding author: Mofeed Al-Nowihi, Microbiology Branch, Department of Biology, Faculty of Science, Sana'a University, Sana'a, Yemen, Tel: 00967774595498; Email: Mofeed.hashem@gmail.com

\section{Research Article}

Volume 3 Issue 2

Received Date: November 29, 2019

Published Date: December 18, 2019

DOI: $10.23880 / j i d t m-16000132$

\section{Abstract}

Background: Because of limited treatment options for brucella infection, preventive strategies are important. Knowledge and awareness are essential for the success of preventive strategies.

Objectives: To investigate the knowledge of human brucellosis among pregnant women.

Study Design: A questionnaire on brucella infection was sent to pregnant women by snowball sampling. Knowledge concerning epidemiology, transmission, symptoms and signs of brucella infection in pregnant women, and treatment options were evaluated.

Results: The questionnaire was completed by 304 pregnant women. The respondents estimated a prevalence of hearing about brucella and brucellosis control of brucella infection varying between $6.3 \%$ to $7.2 \%$.The mean knowledge scores regarding transmission and postnatal symptoms increased with a more advanced career stage (i.e. older age). Gender and parenthood did not contribute to knowledge, but the field of expertise did. Respondents in the field of pediatrics had the highest mean score on postnatal symptoms and long-term effects. Respondents working in the field of gynecology and obstetrics were unaware of the precise transmission route of brucella.

Conclusion: The knowledge of brucella infection among pregnant women in the Netherlands contained several gaps. Increasing knowledge and awareness is expected to enhance the prevention of transmission, to improve recognition, and to stimulate diagnostic investigations and follow-up programs.

Keywords: Seroprevalence; Brucella; Pregnant women; ELISA; Sana'a; Yemen

\section{Abbreviations: ELISA: Enzyme Linked} Immunosorbent Assay; SAT: Standard Agglutination Test. 


\section{Journal of Infectious Diseases \& Travel Medicine}

\section{Introduction}

Brucellosis, known as "undulant fever", "Mediterranean fever" or "Malta fever" is a leading cause of zoonosis worldwide caused by the bacterial genus brucella [1]. Brucella is a small, an aerobic, gram-negative, facultative intracellular, non-motile, non-fermenting, nonspore-forming, cocci, cocobacilli or short rods based on DNA homology and represent a single species [2,3]. Four species cause human diseases which are B. abortus in cattle and buffalo, B. canis in canine, B. suis in pigs, and B. melitensis in sheep, goats and camels which is the most virulent and the principle cause of human infection $[1,4]$. Brucella can be found in both domestic and wild animals. It is transmitted to humans by direct or indirect contact with infected animals or their dairy products $[1,5,6]$. Portals of entry of the organism are the conjunctiva respiratory mucosa and damaged skin [7]. Moreover, the transmission from person to person is uncommon, but the infection from human sources may occur in the following ways: vertical transmission with placental circulation, breast feeding, blood transfusion, bone marrow transplantation and sexual contact [8]. The most common reported symptoms and signs were fever, fatigue, malaise, chills, sweats, headaches, myalgia, and lack of appetite, arthralgia and weight loss [9-12]. The reports on brucellosis in pregnancy published in the literature are [13-15]. However, the first reported was in 1908 when Malta fever, brucellosis, was clinically described [16]. Laterally, on spontaneous abortions in pregnant women could be associated with the isolation of brucella from placenta and aborted fetuses $[17,18]$.

It is believed that brucellosis causes fewer spontaneous abortions in humans than it does in animals because of the absence of erythritol in the human placenta and fetus. Erythritol is a constituent of normal ungulate fetal and placental tissue and, in cases of bovine abortion, promotes overwhelming infection of the placenta and fetus. An additional reason for the lesser role of brucella infection in human abortion is the presence of anti-brucella activity in human amniotic fluid [17]. Brucellosis is an endemic in the South and central Asia, Middle East, North and East Africa, Mediterranean countries of Europe, and central and South America. Worldwide, reported incidence of human brucellosis in endemic disease areas varies widely, from $<0.01$ to $>200$ per 100,000 population [5]. The incidence of human brucellosis in most countries is unknown and it has been estimated that the incidence may be 25 times higher than the reported incidence due to misdiagnosis and underreporting.
Although, Yemen is one of the developing countries where brucellosis remains a major endemic health problem, but there is no published data concerning brucella seroprevalence during pregnancy. It should be performed to contribute in establishing primary database of brucella incidence and prevalence in Yemen. This basic data concerning brucella infection during pregnancy is an important for health planners and care providers. Also, the current study is a trial to detect the effect of brucellosis in pregnancy, awareness and knowledgeable among Yemeni pregnant women. In Yemen, where a large number of animals are kept closed as mixed flocks, there is a relatively lack of information, awareness and knowledge about the epidemiology of brucellosis in livestock, accompanied with an absence of control measures and programs especially in the non-commercial farms. Earlier studies carried out in 1980 revealed that the average brucella prevalence of animal brucellosis was $27 \%$ in goats, and 30\% in sheep [19]. While the first report of the prevalence for human brucellosis was $0.7 \%$ among blood donors [20]. No previous study conducted to determine the prevalence of human brucellosis among pregnant women. All the previous studies conducted in Yemen were either in a limited area and/or period and did not reflect the exact impact of the prevalence of brucellosis among pregnant women in Yemen. So, our study is the first study carried out to revealed the prevalence, measure awareness and knowledgeable of brucella infection among pregnant women.

Moreover, up to date, no systemic research on the epidemiology of brucellosis that cover all the regions of Yemen was carried out to the best of our knowledge, therefore, the objective of this study was undertaken to determine the prevalence of the disease; measure awareness and knowledgeable of brucella infection among pregnant women in Sana'a city, Yemen.

\section{Materials and Methods}

\section{Questionnaire Development}

A questionnaire on brucella, taking less than $4 \mathrm{~min}$ to complete, was developed. The questionnaire tested knowledge of the prevalence of brucella antibodies; the symptoms and signs of brucella in pregnant women. All but four questions regarding knowledge were multiple choices. The possible answers were based on the literature. Multiple answers were accepted. Several demographic variables were asked for, including age, residence, occupation and professional field. When a respondent had not heard of brucella at all, the questionnaire ended after recording the characteristics of 
the respondent. The questionnaire was pilot tested using a convenience sample, and ambiguous questions were rephrased.

\section{Sampling Frame and Questionnaire Administration}

The digital questionnaire, accompanied by a covering letter, was sent to the medical contacts of the researchers. Snowball sampling was used; all participants were asked many questions knowledge about brucella. These contacts were interns, residents, hospital-based senior doctors, general practitioners and medical researchers. In this study the analysis was restricted to the replies of the pregnant women.

\section{Data Analysis}

All statistical tests were carried out using SPSS version 21 (SPSS Inc., Chicago, IL, USA), with the significance levels set at $\mathrm{P}<0.05$. Frequency responses to all questionnaire items were determined, and overall scores were calculated per questionnaire item. This overall score was based on the sum of the correctly stated true answers and the correctly not chosen false answers, assigning one point per correct answer. The maximum achievable score varied between 7 and 12 points, depending on the questionnaire item. Comparisons between different groups of respondents were made using _2 tests.

\section{Questionnaire}

The questionnaire was adapted from a previous questionnaire established and validated by an Arabic group and consisted of 50 questions grouped into five sections. Section 1 intended to evaluate the personal information. Section 2 contained questions about sociodemographic data such as age, residence, and occupation and education level. Section 3 consisted of questions on symptoms. Section 4 contained to awareness and knowledge of brucella. Section 5 contained questions about the pervious diagnosis. The English questionnaire was translated into Arabic, the most frequently spoken languages in our area. The database was created using specific software (Cardiff TeleForm, version 10.2, Cardiff,
Vista, CA, USA) that transfers the data of a scanned questionnaire directly into a database.

\section{Statistical Analysis}

Factors were described by counts and percentage. Participants' characteristics were compared between women with and without knowledge of brucella using chisquared tests or Fisher's exact test. Odds ratios were also reported. Multivariable logistic regression analysis was conducted to assess independent associations between these characteristics and knowledge of brucella. The association between the knowledge of brucella and the practitioners who followed the women during the 1st trimester was assessed with an additional logistic regression model in the subgroup of women with a follow-up during the 1st trimester. Sources of information regarding brucella and preventive measures were compared according to the type of follow-up (midwife, obstetrician or general practitioner) using Fisher's exact test. The proportions of women aware of brucella and other congenital diseases were compared using the McNemar test. Statistical analyses were performed using S-plus 8.0 for Windows (Insightful Corp, Seattle, WA, USA). All statistical tests were two-sided and a p-value < 0.05 was considered as statistically significant.

\section{Results}

\section{Awareness and Knowledge of Brucella}

All serum samples were screened by the SAT and confirmed by ELISA for the presence of antibodies against Brucella antigens (Figure 1). A total of 304 serum sample has been taken from pregnant women. They were tested for brucella antibodies and measure knowledge and awareness for the all subjects. Only 82 of 304 had knowledge and awareness for brucella infection. Of 82 pregnant women 22 had Hear about brucellosis, 20, 19 and 21 had Transmission awareness, Symptoms awareness, Brucellosis control respectively. The level of awareness and knowledge of brucella was very low, despite the fact that the majority of the pregnant women were educated to the tertiary level (Table 1).

\begin{tabular}{|c|c|c|c|}
\hline Category & No. of Samples & No. of Positive & (\%) \\
\hline Hear about brucellosis & 304 & 22 & 7.2 \\
\hline Transmission awareness & 304 & 20 & 6.6 \\
\hline Symptoms awareness & 304 & 19 & 6.3 \\
\hline Brucellosis control & 304 & 21 & 6.9 \\
\hline
\end{tabular}

Table 1: Seroprevalence of brucella infection among pregnant women according to their awareness and knowledge. 


\section{Journal of Infectious Diseases \& Travel Medicine}

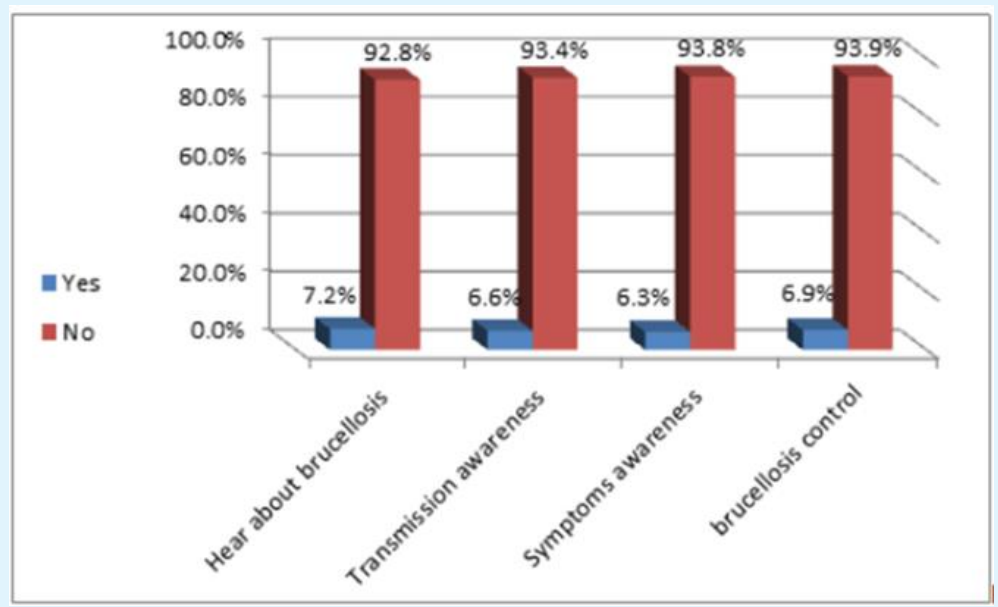

Figure 1: Seroprevalence of knowledge and awareness for human brucellosis in pregnant women.

\section{Discussion}

This is the first published data on the prevalence of brucella infections among pregnant women in Yemen. In this study we found the overall prevalence of brucella antibodies among pregnant 45 (15\%), Education + awareness and knowledge. There was little deviation in prevalence with regard to educational status. Despite the fact that the majority of the pregnant women were educated up to the tertiary level, the level of awareness and knowledge of brucella and its transmission was very low among the study population. This poses a serious problem, as knowledge of a disease and its mode of transmission is important in its prevention and control. With both antibodies, pregnant women who knew about brucella and how it can be transmitted, the infection status of their children, and the availability of possible carriers around them all had a higher prevalence. This poses a serious problem, as it is obvious that knowledge of brucella and its mode of transmission do not prevent infection.

To our knowledge, the results showed that a low prevalence of knowledge and awareness for brucella antibodies in pregnant women. From 304 of the subject 22(7.2\%), 20(6.6\%), 19(6.3\%) and 21(6.9\%) had hear about brucellosis, transmission awareness, Symptoms awareness, and Brucellosis control for brucella antibodies respectively. Therefore, the low of educational level may lead to the low of knowledge and awareness. No previous studies had evaluated the level of knowledge and awareness of brucella infection to compare between their results and our findings either in Yemen or other countries. So, our study was the first study conducted in
Yemen to determine the prevalence of knowledge and awareness for brucellosis among pregnant women.

\section{Conclusion}

The knowledge of brucella infection among pregnant women in Yemen contained several gaps. Increasing knowledge and awareness is expected to enhance the prevention of transmission, to improve recognition, and to stimulate diagnostic investigations and follow-up programs.

\section{Acknowledgement}

We acknowledge contribution of all women in science, who support and contribute in this study. This research work was supported Department of Biology, Faculty of Science, Sana'a University, Sana'a, Yemen. We are also grateful to Al- Thobhani Modern Medical Laboratory, Sana'a, Yemen for their cooperation.

\section{References}

1. Corbel MJ, Elberg SS, Cosivi O (2006) Brucellosis in humans and animals. World Health Organization: 118.

2. Grimont F, Verger JM, Cornelis P, Limet J, Lefèvre M, et al. (1992) Molecular typing of brucella with cloned DNA probes. Res Microbiol 143(1): 55-65.

3. Moreno E, Stackebrandt E, Dorsch M, Wolters J, Busch M, et al. (1990) Brucella abortus 16S rRNA and lipid A reveal a phylogenetic relationship with members of 
the alpha-2 subdivision of the class Proteobacteria. J Bacteriol 172(7): 3569-3576.

4. Lopes LB, Nicolino R, Haddad JPA (2010) Brucellosisrisk factors and prevalence: a review. Vet Sci J 4: 72 84.

5. Boschiroli ML, Foulongne V, O'Callaghan D (2001) Brucellosis a worldwide zoonosis. Curr Opin Microbiol 4(1): 58-64.

6. Chen S, Zhang H, Liu X, Wang W, Hou S, et al. (2014) Increasing threat of brucellosis to low-risk persons in urban settings, China. Emerg Infect Dis 20(1):126130.

7. Mesner O, Riesenberg K, Biliar N, Borstein E, Bouhnik $\mathrm{L}$, et al. (2007) The many faces of human-to-human transmission of brucellosis: congenital infection and outbreak of nosocomial disease related to an unrecognized clinical case. Clin Infect Dis 45(12): 135-140.

8. Peker N, Turan V, Ergenoglu M, Yeniel O (2011) Brucellosis in adolescent pregnancy-case report and review of literature. Ginekol Pol 82(3): 226-229.

9. Ali S, Ali Q, Neubauer H, Melzer F, Elschner M, et al. (2013) Seroprevalence and risk factors associated with brucellosis as a professional hazard in Pakistan. Foodborne Pathog Dis 10(6): 500-505.

10. Dames S, Tonnerre C, Saint S, Jones SR (2005) Clinical problem-solving. Don't know much about history. N Engl J Med 352(22): 2338-2342.
11. Dean AS, Crump L, Greter H, Hattendorf J, Schelling E, et al. (2012) Clinical manifestations of human brucellosis: a systematic review and meta-analysis. PLoS Negl Trop Dis 6(12): 1929.

12. Young EJ (1989) Clinical manifestations of human brucellosis. CRC press, Boca Raton 97: 126.

13. Gulsun S, Aslan S, Satici O, Gul T (2011) Brucellosis in pregnancy. Trop Doct 41(2): 82-84.

14. Khan MY, Mah MW, Memish ZA (2001) Brucellosis in pregnant women. Clin Infect Dis 32(8): 1172-1177.

15. Sharif A, Reyes Z, Thomassen P (1990) Screening for brucellosis in pregnant women. J Trop Med Hyg 93(1): 42-43.

16. Eyre JWH (1908) Melitensis septicemia. Lancet 5: 1747-1752.

17. Seoud M, Saade G, Awar G, Uwaydah M (1991) Brucellosis in pregnancy. J Reprod Med 36(6): 441445.

18. Young EJ (1983) Human brucellosis. Rev Infect Dis 5(5): 821-842.

19. Hosie BD, Al-Bakri OM, Futter RJ (1985) Survey of brucellosis in goats and sheep in the Yemen Arab Republic: comparison of tests for Brucella melitensis infection in sheep. Trop Anim Health Prod 17(2): 9399.

20. Al- Shamahy H (1997) The prevalence of brucella antibodies in Yemen. Saudi Med J 18(1): 45-48. 